\title{
THERMAL MODELING OF THE TEVATRON MAGNET SYSTEM
}

\author{
J. C. Theilacker and A. L. Klebaner \\ Fermi National Accelerator Laboratory* \\ Batavia, IL, 60510, USA
}

\begin{abstract}
Operation of the Tevatron at lower temperatures, for the purpose of allowing higher energies, has resulted in a renewed interest in thermal modeling of the magnet strings. Static heat load and AC loses in the superconducting coils are initially transported through subcooled liquid helium. Heat exchange between the subcooled liquid and a counter flowing two-phase stream transfers the load to the latent heat. Stratification of the twophase helium stream has resulted in considerably less heat exchange compared to the original design. Spool pieces have virtually no heat transfer to the two-phase resulting in a "warm" dipole just downstream. A model of the magnet string thermal behavior has been developed. The model has been used to identify temperature profiles within magnet strings. The temperature profiles are being used in conjunction with initial magnet quench performance data to predict the location of quench limiting magnets within the Tevatron. During thermal cycles of magnet strings, the model is being used to "shuffle" magnets within the magnet string in order to better match the magnets quench performance with its actual predicted temperature. The motivation for this analysis is to raise the operating energy of the Tevatron using a minimal number of magnets from the spares pool.
\end{abstract}

\section{DEFINITIONS}

$\begin{array}{rlrl}\text { Cp } & {[\mathrm{J} / \mathrm{g}-\mathrm{K}]} & = & \text { fluid specific heat } \\ \mathrm{f} & {[-]} & =\text { fraction of the } 1 \varphi \text { flow passing through the } 1 \varphi_{\text {inner }} \text { (coil) passage } \\ \mathrm{f}_{\mathrm{HX}} & {[-]} & = & \text { fraction of the } 1 \varphi_{\text {outer }} \text { mass flow which effectively heat } \\ & & \text { exchanges with the } 2 \varphi \\ \mathrm{m} & {[\mathrm{g} / \mathrm{s}]} & = & \text { total bulk } 1 \varphi \text { mass flow for the magnet string } \\ \varepsilon & {[-]} & = & \text { heat exchanger effectiveness } \\ \mathrm{Ntu} & {[-]} & = & \text { number of heat transfer units }\end{array}$

\footnotetext{
${ }^{*}$ Work supported by the U.S. Department of Energy under contract No. DE-AC02-76CH03000.
} 
$\mathrm{Q}_{1 \varphi-2 \varphi}$ [watts] $=$ distributed heat transfer between the $1 \varphi$ outer and $2 \varphi$ passages

$\mathrm{Q}_{1 \varphi \text { outer }}[$ watts $]=$ distributed static heat load into the $1 \varphi_{\text {outer }}$ passage (predominately through supports)

$\mathrm{Q}_{2 \varphi} \quad[$ watts $]=$ distributed static heat load into the $2 \varphi$ circuit

$\mathrm{Q}_{\mathrm{AC}} \quad[$ watts $]=$ distributed hysteresis and eddy current losses (predominately hysteresis in the superconductor)

$\mathrm{Q}_{\mathrm{ds}} \quad[\mathrm{watts}]=$ static heat load into the $1 \varphi$ at the downstream end of the magnet

$\mathrm{Q}_{\text {net }} \quad[$ watts $]=$ distributed net heat transferred out of the $1 \varphi_{\text {outer }}$ passage

$\mathrm{Q}_{\mathrm{us}} \quad[\mathrm{watts}]=$ static heat load into the $1 \varphi$ at the upstream end of the magnet

$\mathrm{T}_{2 \varphi} \quad[\mathrm{K}]=2 \varphi$ fluid temperature

$\mathrm{T}_{\mathrm{x} 3} \quad[\mathrm{~K}]=1 \varphi$ fluid temperature at the cold end of the exchanger

$\mathrm{T}_{\mathrm{x} 2} \quad[\mathrm{~K}]=1 \varphi$ fluid temperature at the warm end of the exchanger

UA $[\mathrm{watts} / \mathrm{K}]=$ overall heat conductance of the heat exchanger

\section{MODEL}

The Tevatron satellite refrigeration system consists of twenty-four refrigerators, each cooling two $125 \mathrm{~m}$ magnet strings as in FIGURE 1. Each magnet string is typically made up of four optical half cells. A half cell consists of a quadrupole magnet, spool piece and four dipole magnets. The dipoles and quadrupole magnets are of a warm iron design. This results in a relatively high heat leak per magnet. The superconducting coils are cooled by subcooled liquid helium at 2.2 bar. At the end of the $125 \mathrm{~m}$ string, this single-phase subcooled liquid is throttled through a JT valve into two-phase at a pressure determined by the cold compressor operating point and the magnet string two-phase pressure drop.

In FIGURE 1, D, Q, S, T and F stands for dipole, quadrupole, spool piece, turnaround box and feed can, respectively. The magnet string on the left is considered upstream, while the one on the right is considered downstream. Existing instrumentation is also shown as T, P, F and DP for temperature, pressure, flow and differential pressure, respectively. For convenience, the single-phase is shown on the bottom and two-phase on the top, although in actuality, they are mostly concentric with the two-phase on the outside. The interconnect between magnets is not concentric.

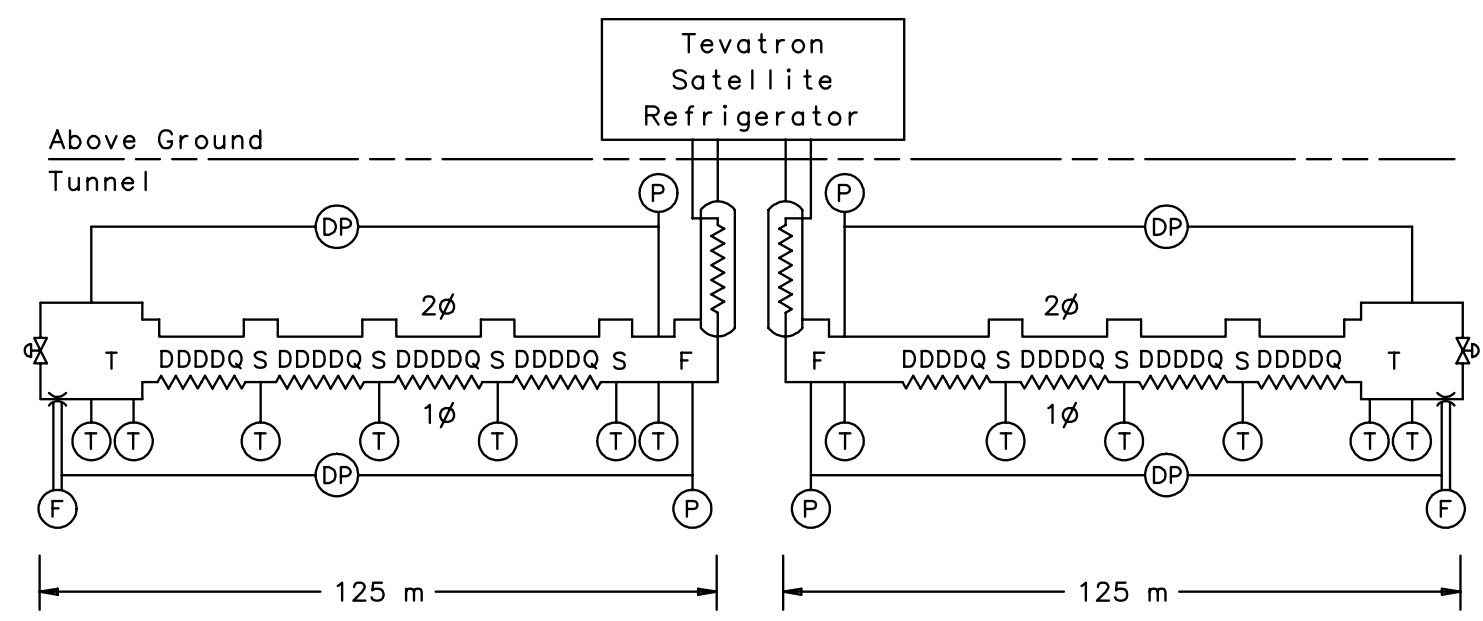

FIGURE 1. Schematic representation of a Tevatron magnet string. 
On its way back to the refrigerator, the two-phase stream counter heat exchanges with the single-phase stream in order to maintain its subcooling. Static and dynamic heat load of the magnet string is therefore absorbed by the latent heat of the two-phase. Single-phase helium acts as a transport media in order to direct heat to the liquid in the two-phase stream. The spool pieces have minimal heat exchange between the single and two-phase helium streams. As a result, the single-phase stream heats up as it passes through the spool.

The quadrupole and dipole magnets have a heat transfer model depicted in FIGURE 2 for a downstream magnet string. X1 through X5 represent station points in the model. $\mathrm{X}$ is incremented from 1 to $\mathrm{n}$ for $\mathrm{n}$ components in the magnet string. Within a magnet, the single-phase flow evenly splits into two streams (inner and outer). The inner stream bathes the superconducting coil. If AC losses are present, as there would be in a ramping Fixed Target physics mode, then this stream increases in temperature as it goes through the magnet. In Collider mode, the stream passes through the magnet with virtually no change.

The purpose of the outer flow stream was to heat exchange with the two-phase and then recombine with the inner stream at the end of the magnet. In practice, it was found that this heat exchange was limited due to stratification of the two-phase stream [2]. As a result, the model further divides the outer stream into an upper and lower stream. Only the lower stream effectively heat exchanges with liquid in the two-phase stream. The lower stream reduces in temperature as it passes through the magnet and approaches the twophase temperature. The upper stream increases in temperature through the magnet due to its share $\left(1-\mathrm{f}_{\mathrm{HX}}\right)$ of the $\mathrm{Q}_{1 \varphi \text { outer }}$ (For clarity, this stream is not shown in FIGURES 3-6.)

The original design of the magnet was intended for the entire heat leak to helium temperatures, except $\mathrm{Q}_{\mathrm{AC}}$, to be intercepted by the two-phase. This was based on the design intention of the two-phase to operate in the froth flow regime. It was later found that the two-phase operates in a highly stratified flow regime [3].

Calculations and flow tests have shown the inner flow split to be 50\% ( $\mathrm{f}=0.5$ ). Geometry of the outer flow passage suggests an effective heat transfer flow split of $50 \%$ $\left(\mathrm{f}_{\mathrm{HX}}=0.5\right)$. For this paper, the model used the following heat loads, in watts, for Tevatron dipoles in Collider mode: $\mathrm{Q}_{\mathrm{AC}}=0, \mathrm{Q}_{\mathrm{us}}=1, \mathrm{Q}_{\mathrm{ds}}=3, \mathrm{Q}_{1 \varphi \text { outer }}=2, \mathrm{Q}_{2 \varphi}=2$. A mass flow rate of $20 \mathrm{~g} / \mathrm{s}$ was used for each magnet string.

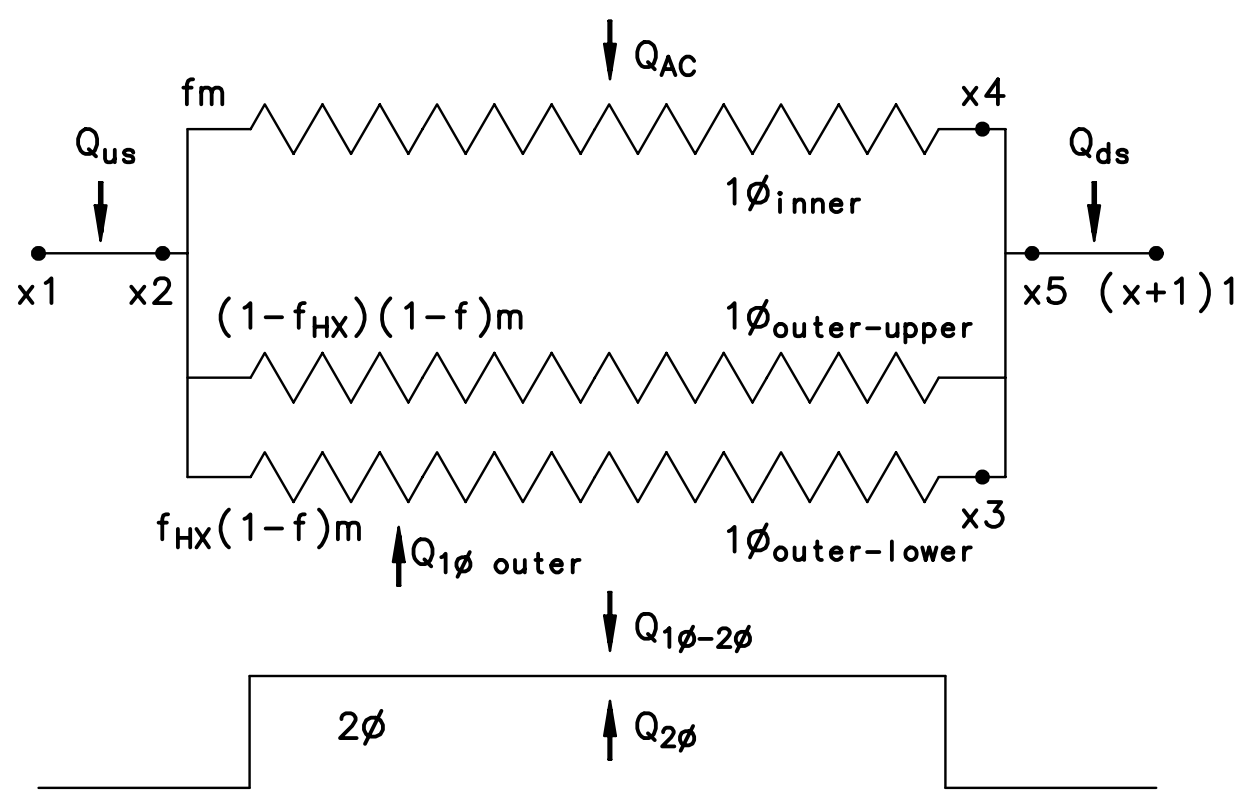

FIGURE 2. Schematic representation of heat transfer in a downstream Tevatron dipole. 
The energy balance for the $1 \varphi_{\text {outer-lower }}$ is given as

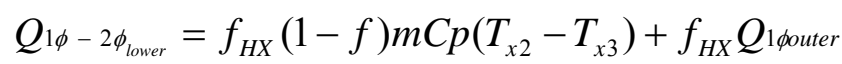

which can be rearranged to

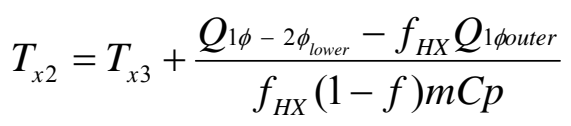

and

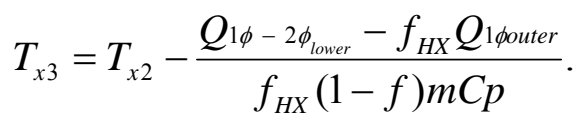

Heat transfer between the $1 \varphi$ outer and $2 \varphi$ can be modeled as an evaporator. Soyars [4] has shown that an evaporator with external heat leak can be modeled using the effectiveness-Ntu method as:

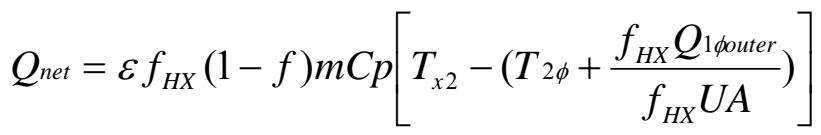

$$
\begin{aligned}
& Q_{\text {net }}=Q_{1 \phi-2 \phi_{\text {lower }}}-f_{H X} Q_{1 \text { pouter }} \\
& Q_{n e t}=f_{H X}(1-f) m C p\left(T_{x 2}-T_{x 3}\right)
\end{aligned}
$$

where,

$$
\varepsilon=1-e^{-N t u}
$$

and

$$
N t u=\frac{f_{H X} U A}{f_{H X}(1-f) m C p} .
$$

Note that the consequence of the external heat leak $\left(\mathrm{Q}_{1 \varphi \text { outer }}\right)$ is to raise the effective $2 \varphi$ temperature by the factor shown in equation (4).

A model, which rigorously considers the convective heat transfer on the single-phase and a superimposed convective and boiling heat transfer on the two-phase, was developed. In order to avoid iterative solutions, a simplified model was also developed when combining the model with the database of quench currents for all 1,000 magnets.

Simple mixing of flow streams or direct addition of heat leak determines the remaining state points. The model for each magnet is then linked together for the entire magnet string. A quadrupole magnet is similar to the dipole with the exception of the single-phase flow split $(\mathrm{f}=0.75)$ and the heat transfer area and distributed heat leaks were scaled to the shorter quadrupole length. 


\section{APPLICATION}

FIGURES 3 and 4 show the resulting temperature profiles of an upstream and downstream magnet string, respectively, for a $900 \mathrm{GeV}$ colliding beam physics run. The refrigerator is on the right side of FIGURE 3 and on the left side of FIGURE 4. The lower line represents the two-phase temperature; the upper line represents the single-phase inner flow and the intermediate curved saw tooth represents the lower single-phase outer flow heat exchanging with the two-phase. Each gridline on the abscissa represents interfaces between components, either a dipole magnet, quadrupole magnet or spool piece.

The two figures point out that the half cell just downstream of the refrigerator (x5 Half Cell) is the coldest, and thus the most favorable location for lower quench energy magnets. FIGURE 4 also shows that the bulk single-phase flow warms as it passes through the $x 5$ half cell. This is due to the heat leak in being higher than can be transferred out at the given $1 \varphi$ to $2 \varphi$ temperature difference. Half cell $x 4$ in FIGURE 3 shows that the break-even temperature difference is about $175 \mathrm{mK}$. For higher temperature differences, the bulk single-phase temperature decreases through the half cell.

The single line connecting half cells represents a spool piece. All helium temperature heat leak to a spool is considered to go directly into the bulk single-phase flow stream. The dot in the middle of the line represents the approximate location of a thermometer. Spool piece thermometers, as well as magnet string inlet and outlet, are the only thermometers in the magnet strings.

The figures show the adverse effect a spool piece heat leak has on the magnet temperature profile. The dipole just downstream (flow direction, away from the refrigerator) of a spool is typically the warmest in the half cell. These locations are generally reserved for higher quench performance magnets. Note that the upstream magnet string begins with a spool piece (right side of FIGURE 3). Otherwise, the $\mathrm{x} 4$ half cell would also be a thermally favorable location.

FIGURES 5 and 6 show the same plots for $980 \mathrm{GeV}$ collider operation in a typical satellite refrigerator. The two-phase temperature was lowered $630 \mathrm{mK}$ using the cold compressor. This resulted in only a $480 \mathrm{mK}$ decrease in peak magnet temperature, due to the reduction in helium specific heat at lower temperatures. Lower specific heat has the effect of exaggerating the temperature profile throughout the string.

The thermal model was combined with the magnet quench database for each of the 1,000 superconducting magnets in the Tevatron. This allowed the individual magnet quench data obtained from our magnet test facility to be scaled to its actual operating temperature. Plotting the resulting scaled quench currents then allows us to quickly identify potentially limiting magnets. We use this technique to aid us in shuffling magnets to better achieve a flat quench profile anytime we warm a pair of magnet strings to room temperature. To date, this technique has been used to shuffle magnets in nine of the twentyfour refrigerators of the Tevatron.

Spare Tevatron magnets are in limited supply and considerable time would be required to restart a production line. As a result, we must ensure that we are using existing magnets in the most effective way possible. To aid in that process and to help guarantee cryogenic margin, ways to locally lower the temperature in the Tevatron are being pursued. The model has been used to locally improve the temperature profile using recooled spool pieces $[5,6]$. This method builds another thermally favorable half cell into the temperature profile as well as improve the profile in half cells downstream. 
Tevatron Upstream Magnet $1 \phi$ Analysis

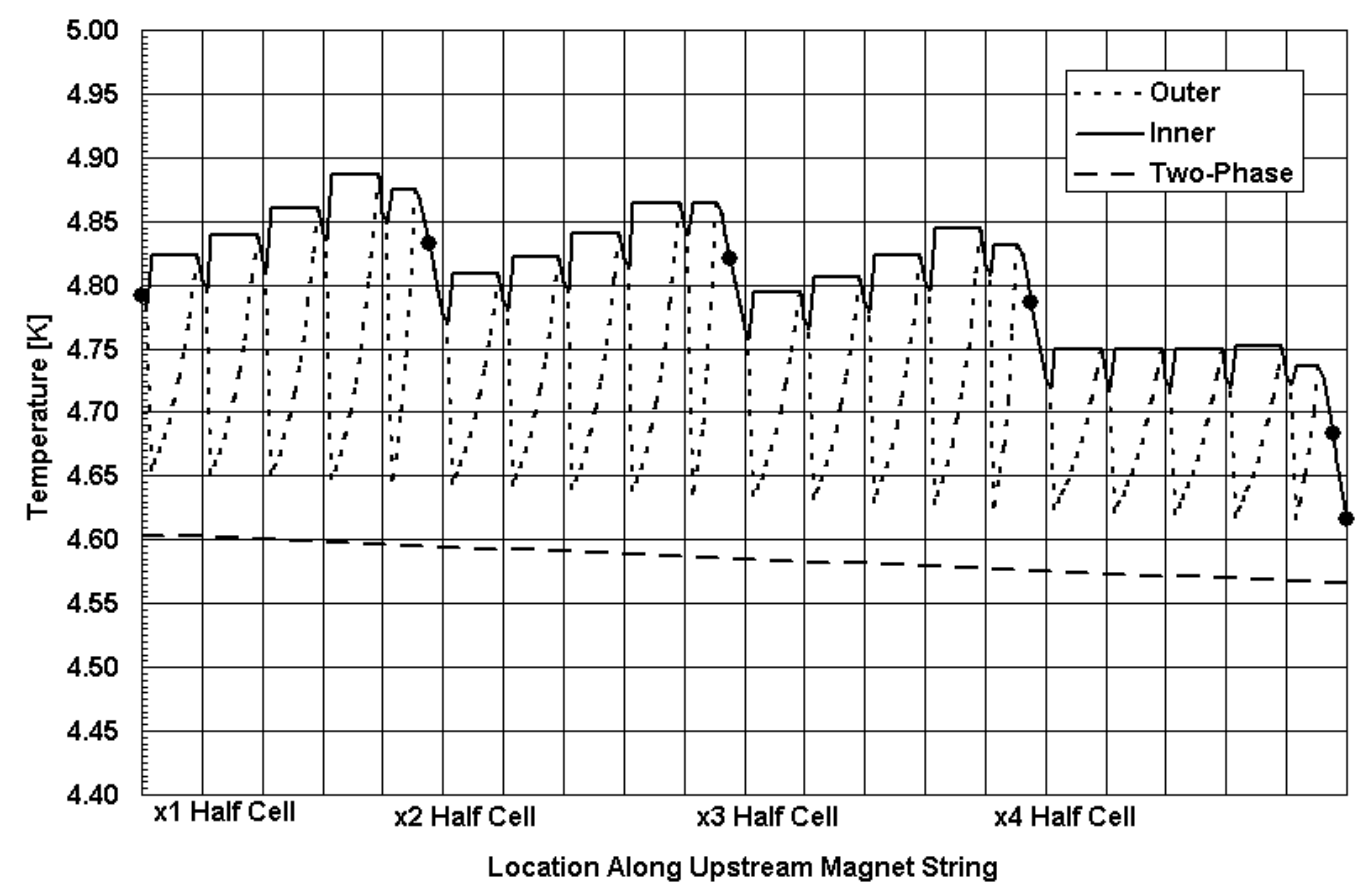

FIGURE 3. Nominal upstream magnet string temperature profile.

Tevatron Downstream Magnet $1 \phi$ Analysis

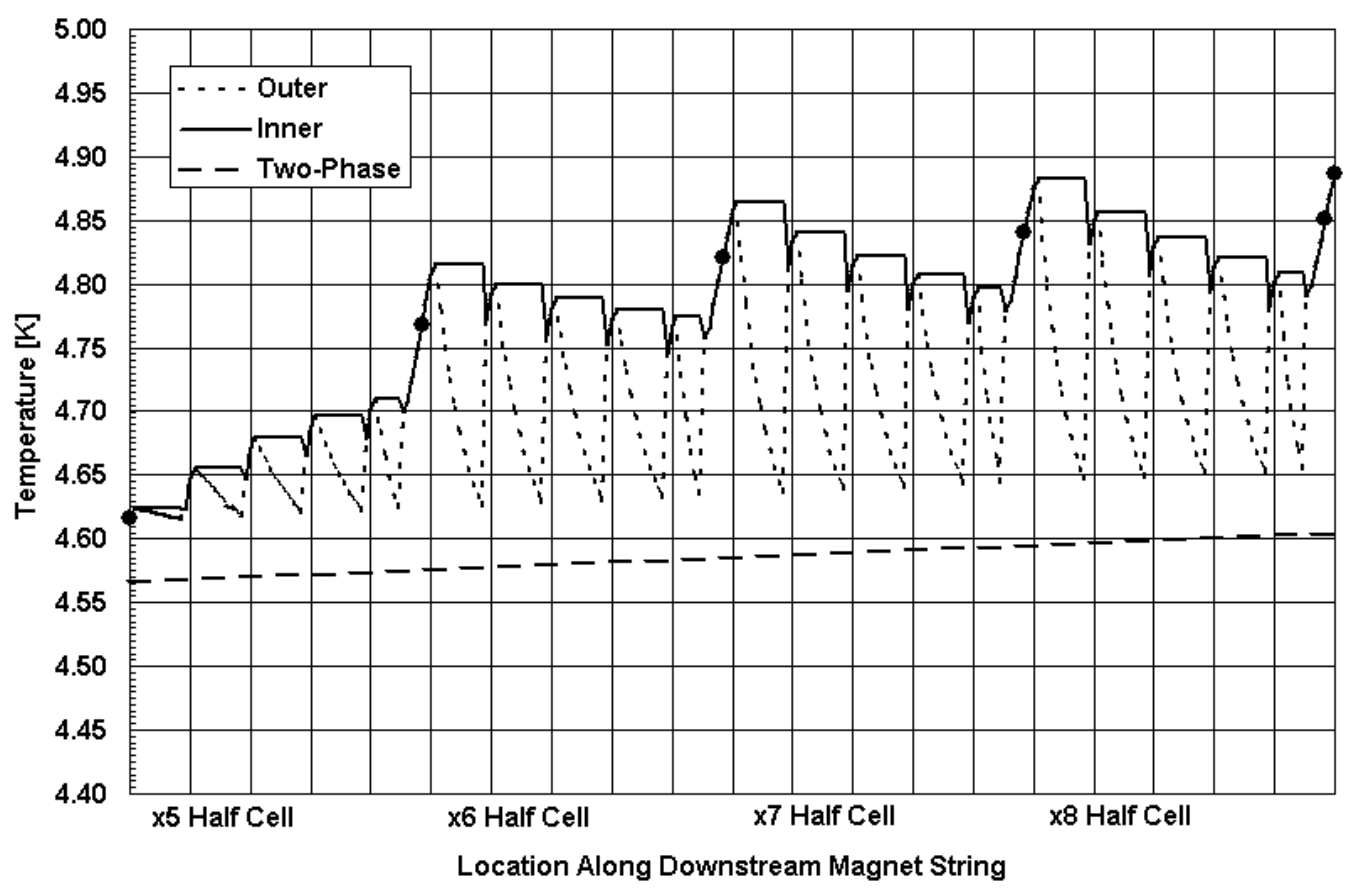

FIGURE 4. Nominal downstream magnet string temperature profile. 


\section{Tevatron Upstream Magnet $1 \phi$ Analysis}

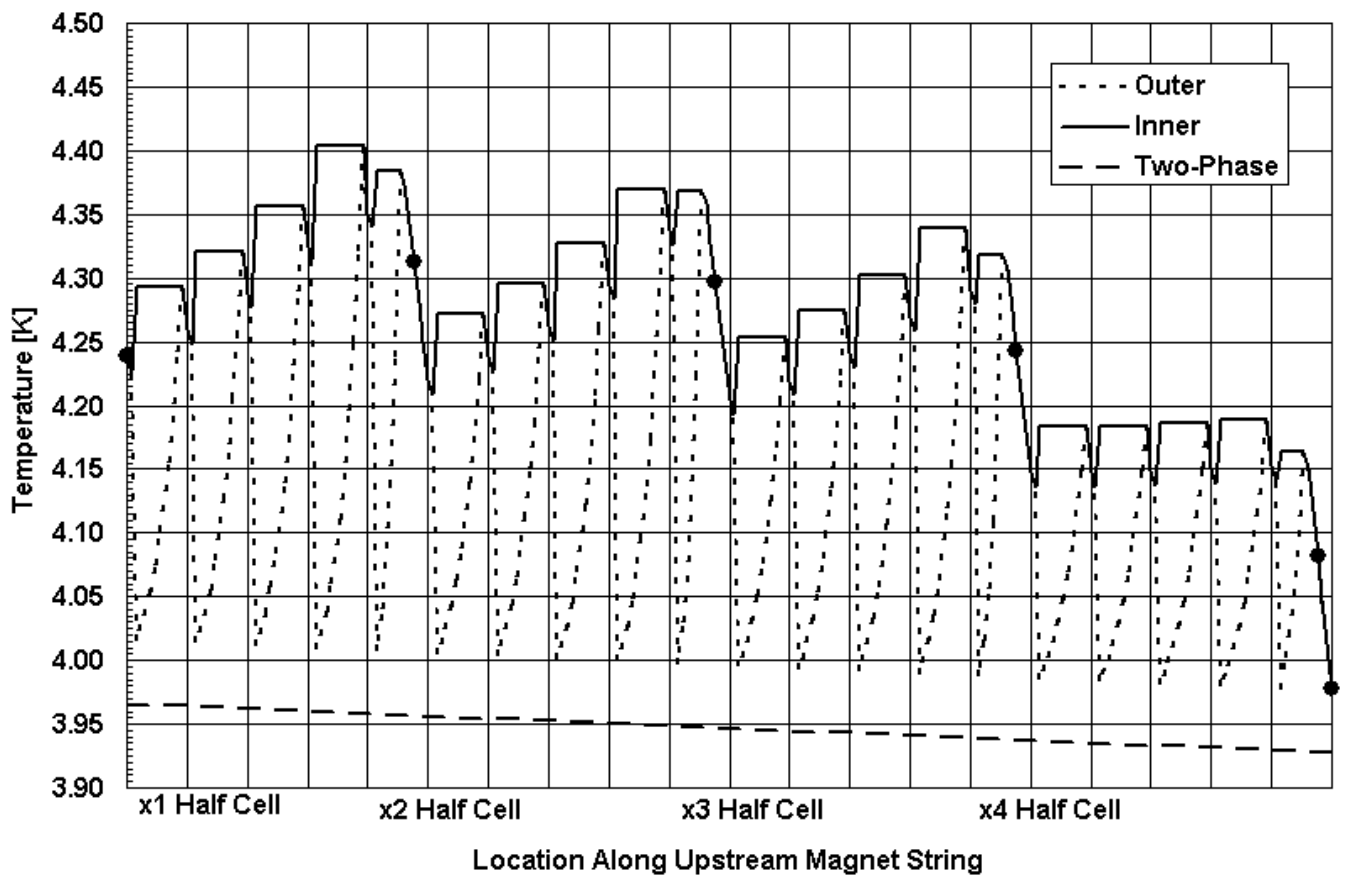

FIGURE 5. Lower temperature upstream magnet string temperature profile.

\section{Tevatron Downstream Magnet $1 \phi$ Analysis}

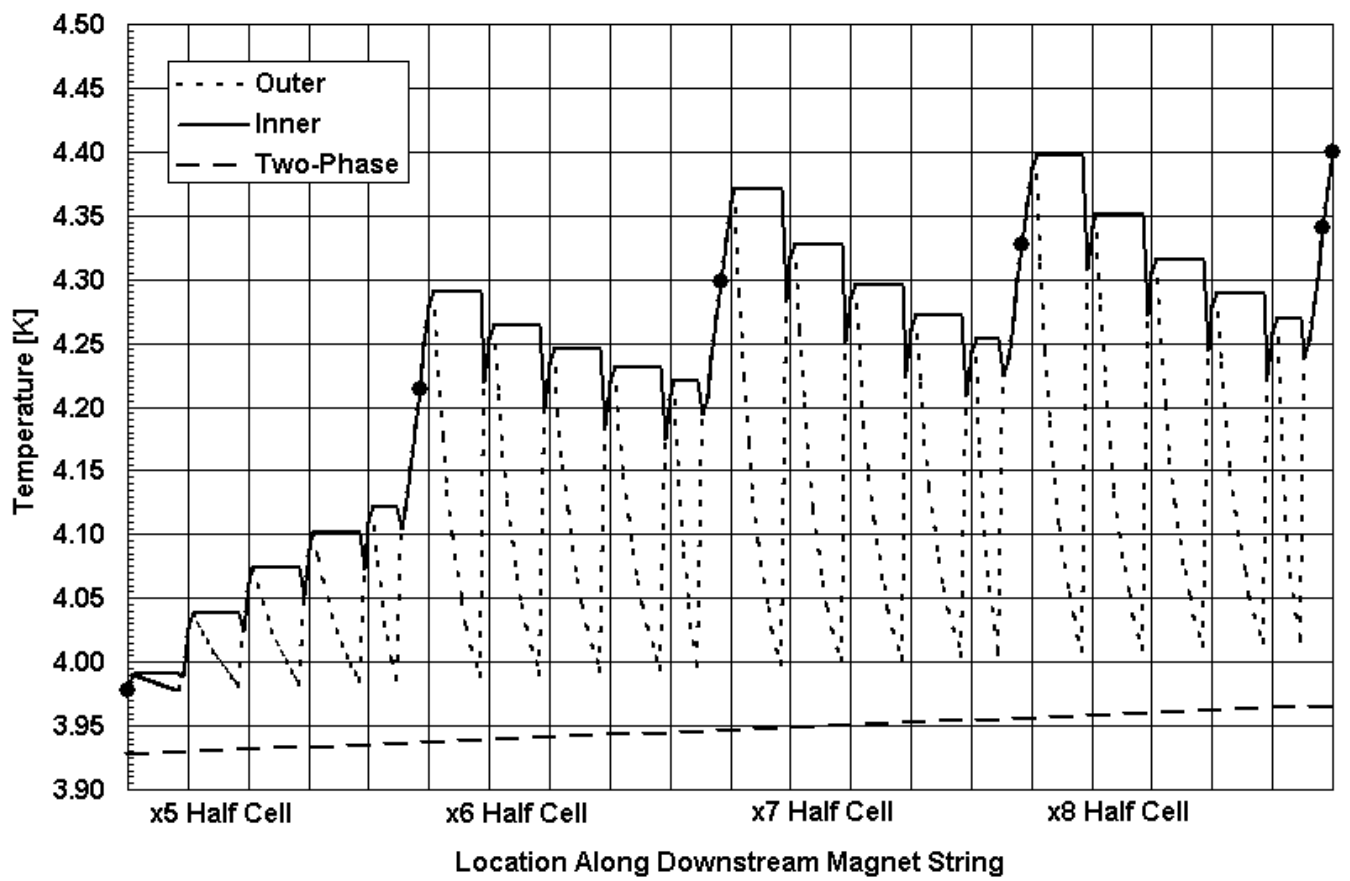

FIGURE 6. Lower temperature downstream magnet string temperature profile. 


\section{CONCLUSIONS}

An effective thermal model of the Tevatron magnet strings has been developed. The model is used to predict the temperature profile throughout the magnet string. When coupled with the individual magnet quench data, the model becomes an effective tool for estimating the limiting magnets in a magnet string. This information is being used to shuffle magnet locations to best match the quench performance with the thermal locations. Improvements in the thermal profile of the magnet strings have been implemented, using the model to predict the performance improvement.

Verification of the model using the Tevatron is very difficult due to the limited thermometry available. Inputs to the model have been refined following the test of a single highly instrumented dipole in our Magnet Test Facility. The model will continue to be improved and enhanced as a tool to optimize the required operating temperature in the Tevatron.

\section{REFERENCES}

1. Theilacker, J. C., Cryogenics 34 ICEC Supplement, pp. 107-110 (1994).

2. Peterson, T. J., Cryogenics 37, pp. 727-732 (1997).

3. Theilacker, J. C. and Rode, C. H., "An Investigation Into Flow Regimes for Two-Phase Helium Flow," in Advances in Cryogenic Engineering 33, edited by R. W. Fast, Plenum Press, New York, 1988, pp. 391398.

4. Soyars, W., "Derivation of $\varepsilon$-NTU Method for Heat Exchangers with Heat Leak," Fermilab Technical Memo 2153.

5. Klebaner, A. L. and Theilacker, J. C., "Single-Phase Helium Recooling In A Tevatron Spool Piece," to be presented at this conference.

6. Klebaner, A. L. and Theilacker, J. C., "Thermal Performance of the Tevatron Magnets Under Higher Energy Operating Conditions," to be presented at this conference. 